\title{
Heisenberg-type higher order symmetries of superintegrable systems separable in Cartesian coordinates
}

\author{
F Güngör ${ }^{1}$, Ş Kuru ${ }^{2}$, J Negro ${ }^{3}$ and L M Nieto ${ }^{3}$ \\ ${ }^{1}$ Department of Mathematics, Faculty of Science and Letters, Istanbul Technical \\ University, 34469 Istanbul, Turkey \\ 2 Department of Physics, Faculty of Science, Ankara University, 06100 Ankara, \\ Turkey \\ 3 Departamento de Física Teórica, Atómica y Óptica, Universidad de Valladolid, \\ 47011 Valladolid, Spain
}

E-mail: gungorf@itu.edu.tr, sengul.kuru@ @science.ankara.edu.tr, jnegro@fta.uva.es and luismiguel.nieto.calzada@uva.es

Received 19 May 2016, revised 5 February 2017

Accepted for publication 3 March 2017

Published 23 March 2017

Recommended by Professor Beatrice Pelloni

\begin{abstract}
Heisenberg-type higher order symmetries are studied for both classical and quantum mechanical systems separable in Cartesian coordinates. A few particular cases of these types of superintegrable systems were already considered in the literature, but here they are characterized in full generality together with their integrability properties. Some of these systems are defined only in a region of $\mathbb{R}^{n}$, and in general they do not include bounded solutions. The quantum symmetries and potentials are shown to reduce to their superintegrable classical analogs in the $\hbar \rightarrow 0$ limit.
\end{abstract}

Keywords: superintegrability, higher order symmetries, quantum superintegrable systems Mathematics Subject Classification numbers: 70S10, 81R12, 70H06

(Some figures may appear in colour only in the online journal)

\section{Introduction}

We will consider a classical two-dimensional Hamiltonian, $H$, separable in Cartesian coordinates having the form 


$$
H=H_{x}+H_{y}, \quad H_{x}=p_{x}^{2}+v_{x}(x), \quad H_{y}=p_{y}^{2}+v_{y}(y) .
$$

As this system is separated, there are two integrals of motion: one of them is the Hamiltonian itself $H$, while the other one, $A$, can be taken, for example, as the difference of both component Hamiltonians, $A=H_{x}-H_{y}$. Therefore, the system is integrable (there are two constants of motion $H$ and $A$ in involution). In this paper, we want to search for systems having this general structure and allowing for another independent constant of motion, $B$, polynomial in the momentum variables $p_{x}, p_{y}$. So, such systems will be superintegrable, with three independent integrals $H, A$ and $B$. We will restrict ourselves to a special class of such superintegrable systems, based on a particular property of the one-dimensional component Hamiltonians, as it is shown below.

First, we want that the additional integral of motion $B_{n}$ for this Hamiltonian be also separable in the coordinates $x, y$ in the form

$$
B_{n}\left(x, y, p_{x}, p_{y}\right)=B_{n x}\left(x, p_{x}\right)-B_{n y}\left(y, p_{y}\right) .
$$

Second, we ask the functions $B_{n x}$ and $B_{n y}$ to be $n$-degree polynomials in the momentum variables $p_{x}, p_{y}$ :

$$
B_{n x}\left(x, p_{x}\right)=\sum_{j=0}^{n} f_{j}(x) p_{x}^{j}, \quad B_{n y}\left(y, p_{y}\right)=\sum_{j=0}^{n} g_{j}(y) p_{y}^{j},
$$

the coefficients $f_{j}(x), g_{j}(y)$ being some unknown functions, depending on the variables $x$ and $y$, respectively.

Third, the functions $B_{n x}$ and $B_{n y}$ must satisfy the following Heisenberg-type Poisson brackets $(\mathrm{PB})$ :

$$
\left\{H_{x}, B_{n x}\left(x, p_{x}\right)\right\}=\left\{H_{y}, B_{n y}\left(y, p_{y}\right)\right\}=\text { constant }=1 .
$$

The constant can be taken, without loss of generality, equal to one. Then, it is clear that the function $B_{n}$ given by (1.2) will satisfy, together with the Hamiltonian (1.1), the following PB,

$$
\left\{H, B_{n}\left(x, y, p_{x}, p_{y}\right)\right\}=\left\{H_{x}, B_{n x}\left(x, p_{x}\right)\right\}-\left\{H_{y}, B_{n y}\left(y, p_{y}\right)\right\}=0 .
$$

In this way, we have arrived to an 'extra' constant of motion to achieve superintegrability. Such a constant of motion is called to be of Heisenberg type, since it is based on the Heisenberg algebra (1.4) for each of the one dimensional components: $\left\langle H_{x}, B_{n x}, 1\right\rangle$ and $\left\langle H_{y}, B_{n y}, 1\right\rangle$. Each one dimensional Hamiltonian $H_{x}, H_{y}$ is called Heisenberg Hamiltonian. Depending on the value of $n$ we will speak of $n$-degree superintegrable system, when $n \geqslant 3$ the constant of motion $B_{3}$ is said to be of 'higher order' (since the 'standard' constants of motions are of degree two). Recall that the PB for the functions $F\left(x, y, p_{x}, p_{y}\right), G\left(x, y, p_{x}, p_{y}\right)$ is defined in the usual way,

$$
\{F, G\}=\frac{\partial F}{\partial x} \frac{\partial G}{\partial p_{x}}+\frac{\partial F}{\partial y} \frac{\partial G}{\partial p_{y}}-\frac{\partial F}{\partial p_{x}} \frac{\partial G}{\partial x}-\frac{\partial F}{\partial p_{y}} \frac{\partial G}{\partial y} .
$$

We will see that some of the superintegrable Hamiltonians are only defined in regions of the plane $\mathbb{R}^{2}$, furthermore the corresponding potentials will not allow for a bounded motion. Therefore, the evolution of a particle in these potentials will have only a piece of its trajectory in the domain of superintegrability. We could 'extend' any such Hamiltonian to another one defined in the whole plane, but it will not be superintegrable anymore, and in case this new extended Hamiltonian has a bounded motion, in general this motion will not be periodic.

This program can also be carried out for the corresponding quantum systems in a quite similar way. In the quantum context, we write the Hamiltonian operator in the form 


$$
\mathcal{H}=\mathcal{H}_{x}+\mathcal{H}_{y}, \quad \mathcal{H}_{x}=P_{x}^{2}+V_{x}(X), \quad \mathcal{H}_{y}=P_{y}^{2}+V_{y}(Y),
$$

where $P_{x}, P_{y}$ and $X, Y$ are the momentum and position operators, satisfying the well known commutation relations

$$
\left[X, P_{x}\right]=\mathrm{i} \hbar, \quad\left[Y, P_{y}\right]=\mathrm{i} \hbar .
$$

We will work in the coordinate representation where the action of the momentum operators is given by $P_{x}=-\mathrm{i} \hbar \partial_{x}, P_{y}=-\mathrm{i} \hbar \partial_{y}$, and the action of the position operators $X, Y$ is just the multiplication by the variables $x$ and $y$, respectively. This two dimensional Hamiltonian operator (1.7) can be considered integrable in the sense that it has already two independent symmetry operators in involution: $\mathcal{H}$ itself and (for example) $\mathcal{A}=\mathcal{H}_{x}-\mathcal{H}_{y}$. In order to get quantum superintegrable systems of Heisenberg type, as in the classical case, we will look for a symmetry operator, polynomial of degree $n$ in the momentum operators $P_{x}, P_{y}$, having the separated form

$$
\mathcal{B}_{n}\left(X, Y, P_{x}, P_{y}\right)=\mathcal{B}_{n x}\left(X, P_{x}\right)-\mathcal{B}_{n y}\left(Y, P_{y}\right)
$$

where

$$
\mathcal{B}_{n x}\left(X, P_{x}\right)=\sum_{j=0}^{n} f_{j}(X) P_{x}^{j}, \quad \mathcal{B}_{n y}\left(Y, P_{y}\right)=\sum_{j=0}^{n} g_{j}(Y) P_{y}^{j} .
$$

We will ask the component operators to satisfy Heisenberg-type commutation relations,

$$
\left[\mathcal{H}_{x}, \mathcal{B}_{n x}\left(X, P_{x}\right)\right]=\left[\mathcal{H}_{y}, \mathcal{B}_{n y}\left(Y, P_{y}\right)\right]=\mathrm{i} \hbar,
$$

so that the symmetry condition

$$
\left[\mathcal{H}, \mathcal{B}_{n}\right]=0
$$

is automatically satisfied. This symmetry is of order $n$, and when $n \geqslant 3$ it is said to be of 'higher order'.

For a wide discussion of general third and fourth-order integrals of motion, the reader is referred to the excellent review [1]. The one dimensional case of higher order symmetries has been studied in [2, 3]. It is also worth to mention references [4-8], dealing with higher order symmetries, which are more related to our approach. In the Conclusions we will comment on the connection between the methods and results of these references and those obtained in the present paper.

\section{Heisenberg-type higher order integrals of motion: the classical problem}

In this section we will investigate the existence of classical potentials and integrals of motion satisfying equation (1.4) and we will try to determine their explicit expressions. Here, we need to consider only one pair of the variables (for instance $x, p_{x}$ ), because the results are the same for the other variable pair. Also, in order to simplify the notation, we will take $p_{x} \equiv p$, $B_{n x}(x, p) \equiv B_{n}, H_{x} \equiv H_{n}$, and therefore $v_{x}(x) \equiv v_{n}(x)$.

Notice that the PB relation (1.4) can be interpreted as follows. We can think of the Heisenberg function $-B_{n}$ and the Hamiltonian $H_{n}$ as new canonical variables $\tilde{x}, \tilde{p}$ :

$$
\tilde{H}_{n} \equiv \tilde{p}=H_{n}(x, p), \quad \tilde{x}=-B_{n}(x, p) .
$$

The new momentum $\tilde{p}$ is also identified with the new Hamiltonian $\tilde{H}_{n}$. This means that the new pair of canonical variables $\tilde{x}, \tilde{p}$ corresponds to the characteristic function of Hamilton-Jacobi theory [9]. We can solve the motion for the new variables: 


$$
\begin{array}{ll}
\dot{\tilde{x}}=\frac{\partial \tilde{H}}{\partial \tilde{p}}=1, & \tilde{x}=t+\alpha, \\
\dot{\tilde{p}}=-\frac{\partial \tilde{H}}{\partial \tilde{x}}=0, & \tilde{p}=\beta,
\end{array}
$$

where $\alpha, \beta$ are constants fixed by the initial conditions. From the motion of $\tilde{x}, \tilde{p}$, we can find the evolution of the initial variables $x, p$ algebraically by reverting the relations (2.1). In summary, the problem of finding a Heisenberg system characterized by the function $B_{n}$ and Hamiltonian $H_{n}$ is equivalent to the search of systems such that the canonical variables of the characteristic function include the coordinate $\tilde{x}$ given by a polynomial function of degree $n$ in the momentum $p$.

We start this section with a list of particular cases for some values of $n$ in order to see some features of the potentials $v_{n}(x)$ and the functions $B_{n}$. Later on, closed formulas for the general $n$-order case are supplied. Finally, it is explained how the superintegrable systems are obtained together with their properties from the previous results.

\subsection{Particular cases}

- Case $n=1$

The $x$-Hamiltonian and the $x$-part of the integral of motion have the form

$$
H_{1}=p^{2}+v_{1}(x), \quad B_{1}=f_{0}(x)+f_{1}(x) p,
$$

and they must satisfy

$$
\left\{H_{1}, B_{1}\right\}=1 .
$$

Substituting (2.3) in (2.4) we get a set of differential equations from the coefficients of the powers $p^{j}, j=0,1,2$ :

$$
2 f_{1}^{\prime}=0, \quad 2 f_{0}^{\prime}=0, \quad 1=f_{1} v_{1}^{\prime}
$$

where the prime denotes the derivative with respect to the argument. For the sake of simplicity, from now on we omit the explicit dependence of the functions $f_{j}$ and $v_{1}(x)$ on the variable $x$. Thus, for three unknown functions $f_{0}, f_{1}$ and $v_{1}(x)$, there are three equations given by (2.5). The first two equations give $f_{1}=k_{1}$ and $f_{0}=k_{0}$, where $k_{1}$ and $k_{0}$ are integration constants. Thus, from the last equation the potential is

$$
v_{1}(x)=\frac{x}{k_{1}}+c_{1}
$$

where $c_{1}$ is an irrelevant integration constant. Hence, $B_{1}$ takes the form:

$$
B_{1}=k_{0}+k_{1} p .
$$

- Case $n=2$

For this case the function $B_{2}$ is quadratic

$$
B_{2}=f_{0}+f_{1} p+f_{2} p^{2},
$$

and together with $\mathrm{H}_{2}$ satisfy 


$$
\left\{H_{2}, B_{2}\right\}=1 .
$$

Using the Hamiltonian function and (2.8) in (2.9), we obtain a set of equations

$$
2 f_{2}^{\prime}=0, \quad 2 f_{1}^{\prime}=0, \quad 2 f_{0}^{\prime}=2 f_{2} v_{2}^{\prime}, \quad 1=f_{1} v_{2}^{\prime} .
$$

This case lead us to the same potential:

$$
v_{2}(x)=v_{1}(x)=\frac{x}{k_{1}}+c_{1} .
$$

The Heisenberg function $B_{2}$ takes the form

$$
B_{2}=k_{0}+k_{1} p+k_{2} v_{2}+k_{2} p^{2}=k_{0}+k_{1} p+k_{2} H_{2}=B_{1}+k_{2} H_{2} .
$$

Thus, $B_{2}$ is the same as $B_{1}$, except for a 'trivial term' proportional to the Hamiltonian corresponding to the constant $k_{2}$.

- Case $n=3$

For this case the function $B_{3}$ is cubic

$$
B_{3}=f_{0}+f_{1} p+f_{2} p^{2}+f_{3} p^{3} .
$$

After imposing the condition $\left\{H_{3}, B_{3}\right\}=1$, the coefficients $f_{j}$ in (2.13) are the solutions of the set of differential equations

$$
\begin{aligned}
2 f_{3}^{\prime} & =0, \\
2 f_{2}^{\prime} & =0, \\
2 f_{1}^{\prime} & =3 f_{3} v_{3}^{\prime}, \\
2 f_{0}^{\prime} & =2 f_{2} v_{3}^{\prime}, \\
1 & =f_{1} v_{3}^{\prime} .
\end{aligned}
$$

If we solve this system for the functions $f_{j}$, we get the following differential equation for potential

$$
\left(k_{1}+\frac{3}{2} k_{3} v_{3}\right) v_{3}^{\prime}=1
$$

which gives

$$
k_{1} v_{3}+\frac{3}{4} k_{3} v_{3}^{2}=x+c_{3} .
$$

The solution of this quadratic equation can be given explicitly,

$$
v_{3}(x)=\frac{1}{3 k_{3}}\left(-2 k_{1} \pm 2 \sqrt{3 k_{3} x+k_{1}^{2}+3 k_{3} c_{3}}\right),
$$

where $c_{3}$ is an integration constant. The new information for this case is obtained taking $k_{1}=0$ in (2.17). For instance, if we choose $k_{1}=0, k_{3}= \pm 4 / 3, c_{3}=0$ we will have the particular solutions:

$$
v_{3}(x)= \pm \sqrt{ \pm x} .
$$

Remark that depending on the sign, this potential makes sense either for $x \geqslant 0$ or for $x \leqslant 0$. The expression of $B_{3}$ is 


$$
\begin{aligned}
B_{3} & =k_{0}+k_{2} v_{3}+\left(k_{1}+\frac{3}{2} k_{3} v_{3}\right) p+k_{2} p^{2}+k_{3} p^{3} \\
& =B_{1}+k_{2} H_{3}+k_{3}\left(p^{3}+\frac{3}{2} v_{3} p\right)=k_{0}+k_{1} p+k_{2} H_{3}+k_{3} \frac{1}{2}\left(p^{3}-3 H_{3} p\right) .
\end{aligned}
$$

Therefore, we notice that the potential $v_{3}(x)$ depends on three constants: $k_{1}, k_{3}$ and $c_{3}$, but the last constant is irrelevant since it can be eliminated by a translation in $x$. The integral of motion $B_{3}$ depends on the corresponding constants, $k_{1}$ and $k_{3}$; it also includes one additional term proportional to the Hamiltonian, $\mathrm{k}_{2} \mathrm{H}_{3}$, but this can be eliminated without any consequence.

- Case $n=4$

This leads us to the same potential as in the previous case $n=3 ; v_{3}(x)=v_{4}(x)$. But, the function $B_{4}$ is slightly different:

$$
\begin{aligned}
B_{4} & =k_{0}+k_{2} v_{4}+k_{4} v_{4}^{2}+\left(k_{1}+\frac{3}{2} k_{3} v_{4}\right) p+\left(k_{2}+2 k_{4} v_{4}\right) p^{2}+k_{3} p^{3}+k_{4} p^{4} \\
& =B_{1}+k_{2} H_{4}+k_{3}\left(p^{3}+\frac{3}{2} v_{4} p\right)+k_{4}\left(H_{4}\right)^{2} .
\end{aligned}
$$

- Case $n=5$

Now $B_{5}$ is a fifth-order polynomial in $p$

$$
B_{5}=f_{0}+f_{1} p+f_{2} p^{2}+f_{3} p^{3}+f_{4} p^{4}+f_{5} p^{5} .
$$

Imposing the condition $\left\{H_{5}, B_{5}\right\}=1$, the functions $f_{j}$ in $(2.21)$ turn out to be the solutions of the set of differential equations

$$
\begin{aligned}
2 f_{5}^{\prime} & =0, \\
2 f_{4}^{\prime} & =0, \\
2 f_{3}^{\prime} & =5 f_{5} v_{5}^{\prime}, \\
2 f_{2}^{\prime} & =4 f_{4} v_{5}^{\prime}, \\
2 f_{1}^{\prime} & =3 f_{3} v_{5}^{\prime}, \\
2 f_{0}^{\prime} & =2 f_{2} v_{5}^{\prime}, \\
1 & =f_{1} v_{5}^{\prime} .
\end{aligned}
$$

After solving this system for the functions $f_{j}$, the equation for the potential has the form

$$
\left(k_{1}+\frac{3}{2} k_{3} v_{5}+\frac{15}{8} k_{5} v_{5}^{2}\right) v_{5}^{\prime}=1
$$

or

$$
k_{1} v_{5}+\frac{3}{4} k_{3} v_{5}^{2}+\frac{5}{8} k_{5} v_{5}^{3}=x+c_{5}
$$

from which the solution can be explicitly obtained. For instance, if we concentrate on the particular values $k_{1}=k_{3}=c_{1}=0, k_{5}=8 / 5$, we get

$$
v_{5}(x)=\sqrt[3]{x} .
$$


For this case, the Heisenberg function $B_{5}$ is

$$
\begin{aligned}
B_{5} & =k_{0}+k_{4} v_{5}+\left(k_{1}+\frac{3}{2} k_{3} v_{5}+\frac{15}{8} k_{5} v_{5}^{2}\right) p+\left(k_{2}+2 k_{4} v_{5}\right) p^{2}+\left(k_{3}+\frac{5}{2} k_{5} v_{5}\right) p^{3} \\
& +k_{4} p^{4}+k_{5} p^{5} \\
& =k_{0}+k_{1} p+k_{2} H_{5}+k_{3}\left(p^{3}+\frac{3}{2} v_{5} p\right)+k_{4}\left(H_{5}\right)^{2}+k_{5}\left(p^{5}+\frac{5}{2} v_{5} p^{3}+\frac{15}{8} v_{5}^{2} p\right) .
\end{aligned}
$$

We see that the potential $v_{5}(x)$ given in equation (2.24) depends on the constants $k_{1}, k_{3}$ and $k_{5}$ (the constant $c_{5}$ can be eliminated as before by means of a translation). With respect to the function $B_{5}$ it depends on these three constants (the terms including $k_{4}, k_{2}$ and $k_{0}$ can be omitted). The coefficients of these constants have the same expressions in terms of $v(x)$ as the corresponding ones in the previous cases, except for the new one corresponding to $k_{5}$ :

$$
k_{5} B_{5}^{0} \equiv k_{5}\left(p^{5}+\frac{5}{2} v_{5} p^{3}+\frac{15}{8} v_{5}^{2} p\right)=k_{5} \frac{3}{8}\left(p^{5}-\frac{10}{3} p^{3} H_{5}+5 p H_{5}^{2}\right) .
$$

- Case $n=6$

The case $n=6$, gives us the same equation for potential and therefore the same potential as in the previous case $n=5: v_{5}(x)=v_{6}(x)$. The function $B_{6}$ differs from $B_{5}$ in a trivial term proportional to $H_{6}^{3}$.

- Case $n=7$

The integral of motion will be

$$
B_{7}=f_{0}+f_{1} p+f_{2} p^{2}+f_{3} p^{3}+f_{4} p^{4}+f_{5} p^{5},
$$

the coefficients satisfying the system

$$
\begin{aligned}
2 f_{7}^{\prime} & =0, \\
2 f_{6}^{\prime} & =0, \\
2 f_{5}^{\prime} & =7 f_{7} v_{7}^{\prime}, \\
2 f_{4}^{\prime} & =6 f_{6} v_{7}^{\prime}, \\
2 f_{3}^{\prime} & =5 f_{5} v_{7}^{\prime}, \\
2 f_{2}^{\prime} & =4 f_{4} v_{7}^{\prime}, \\
2 f_{1}^{\prime} & =3 f_{3} v_{7}^{\prime}, \\
2 f_{0}^{\prime} & =2 f_{2} v_{7}^{\prime}, \\
1 & =f_{1} v_{7}^{\prime} .
\end{aligned}
$$

The solution of these equations lead to

$$
\left(k_{1}+\frac{3 ! !}{2} k_{3} v_{7}+\frac{5 ! !}{2^{2}} k_{5} \frac{v_{7}^{2}}{2 !}+\frac{7 ! !}{2^{3}} k_{7} \frac{v_{7}^{3}}{3 !}\right) v_{7}^{\prime}=1
$$

or

$$
k_{1} v_{7}+\frac{3 ! !}{2} k_{3} \frac{v_{7}^{2}}{2 !}+\frac{5 ! !}{2^{2}} k_{5} \frac{v_{7}^{3}}{3 !}+\frac{7 ! !}{2^{3}} k_{7} \frac{v_{7}^{4}}{4 !}=x+c_{7},
$$


which is an implicit expression of the potential. The new relevant information in this equation is obtained for $k_{7} \neq 0$. In particular if $k_{1}=k_{3}=k_{5}=c_{7}=0$ and $k_{7}= \pm \frac{2^{3} 4 !}{7 ! !}$, we have the potential:

$$
v_{7}(x)= \pm \sqrt[4]{ \pm x}
$$

Remark that depending on the sign of $k_{7}$ this potential makes sense either for $x \geqslant 0$ or for $x \leqslant 0$.

\subsection{General case}

The functions $B_{n}$ found above can be expressed in two ways: (a) collecting the terms by the integration constants $k_{j}$, or (b) grouping terms in powers of $p$.

(a) Solutions in terms of $k_{i}$

We have seen that the problems for consecutive odd and even degrees $n=2 \ell+1$ and $n=2 \ell+2$ have essentially the same solutions. Therefore, let us restrict to an odd function $B_{n}, n=2 \ell+1, \ell=0,1, \ldots$, and the corresponding potential $v_{2 \ell+1}(x)$, then their expressions take the general form

$$
B_{2 \ell+1}=\sum_{j=0}^{\ell} k_{2 j+1} b_{2 j+1}(p), \quad \sum_{j=0}^{j=\ell} k_{2 j+1} \alpha_{2 j+1} v_{2 \ell+1}^{j+1}=x+c_{2 \ell+1},
$$

where $b_{2 j+1}(p)$ are polynomials of degree $2 j+1$ of $p$ and $\alpha_{2 j+1}$ are constants which are obtained in the integration process, while $k_{2 j+1}$ and $c_{2 \ell+1}$ are arbitrary integration constants. Thus, only the odd integration constants are important, the even ones do not play any role in the solutions.

There are two particular cases worth to mention.

(i) If $k_{2 \ell+1}=0$, but $k_{j} \neq 0, \forall j<\ell$, then the formulas (2.33) valid for $n=2 \ell+1$ come into the ones for the previous case $n=2(\ell-1)+1$. In other words, the formulas for $n=2 \ell+1$, include as particular cases all the formulas for the previous cases.

(ii) If $k_{2 \ell+1} \neq 0$, but $k_{j}=0, \forall j<\ell$, then we get that the potential is a root,

$$
v_{2 \ell+1}(x)=(a x+b)^{1 /(\ell+1)},
$$

where $a, b$ depend on the integration constants, while $B_{2 \ell+1}=k_{2 \ell+1} b_{2 \ell+1}(p)$ is a polynomial of degree $2 \ell+1$, according to (2.33). In conclusion, we can say that this type of superintegrable potentials include all the roots $v_{2 \ell+1}(x) \propto x^{1 /(\ell+1)}$, starting with the trivial linear potential $v_{1}(x) \propto x$.

(b) Solutions in powers of $p$

Now, we will deal with the general case of the polynomial function expressed in powers of $p$ as

$$
B_{n}(x, p)=\sum_{j=0}^{n} f_{j}(x) p^{j}
$$

and substitute it in the PB equation (1.4). Then we get a list of equations for the coefficients $f_{j}$ and the potential $v(x)$, 


$$
\begin{aligned}
2 f_{n}^{\prime} & =0, \\
2 f_{n-1}^{\prime} & =0, \\
2 f_{n-2}^{\prime} & =n f_{n} v^{\prime}, \\
2 f_{n-3}^{\prime} & =(n-1) f_{n-1} v^{\prime}, \\
2 f_{n-4}^{\prime} & =(n-2) f_{n-2} v^{\prime}, \\
\vdots & \vdots \\
2 f_{0}^{\prime} & =2 f_{2} v^{\prime}, \\
1 & =f_{1} v^{\prime} .
\end{aligned}
$$

Remark that for every value of $n$ this system can be completely separated into two: one only for the odd-index $\left(f_{1}, f_{3}, \ldots\right)$ functions and another only for the even-index functions $\left(f_{0} . f_{2}, \ldots\right)$.

We introduce the following notation

$B_{n}(x, p)=f_{\frac{n}{2}, 0} p^{n}+f_{\frac{n-1}{2}, 0} p^{n-1}+f_{\frac{n}{2}, 1} p^{n-2}+f_{\frac{n-1}{2}, 1} p^{n-3}+\cdots+ \begin{cases}f_{\frac{n}{2}, \frac{n}{2}}, & \text { for } n \text { even } \\ f_{\frac{n-1}{2}, \frac{n-1}{2}}, & \text { for } n \text { odd }\end{cases}$

where the 'old' $f_{j}$ and 'new' $f_{\mu, \nu}$ coefficients are related as follows:

$f_{n}=f_{\frac{n}{2}, 0}, \quad f_{n-1}=f_{\frac{n-1}{2}, 0}, \quad f_{n-2}=f_{\frac{n}{2}, 1}, \quad f_{n-3}=f_{\frac{n-1}{2}, 1}, \ldots$

Then, equation (2.36) can be integrated and the coefficients $f_{\mu, \nu}$ are given by

$$
f_{\mu, \nu}=\sum_{\nu^{\prime}=0}^{\nu} \frac{\Gamma\left(\mu+1-\nu^{\prime}\right)}{\Gamma(\mu+1-\nu)} \frac{\nu^{\nu-\nu^{\prime}}}{\left(\nu-\nu^{\prime}\right) !} k_{2\left(\mu-\nu^{\prime}\right)} .
$$

As only the odd index cases seem to be relevant, let us assume that $n=2 \ell+1$ is odd. Then if we substitute the formula for $f_{1}$ given by the last equation of (2.36), we will get

$$
f_{1} v^{\prime} \equiv f_{\ell+\frac{1}{2}, \ell} v^{\prime}=\left(\sum_{\nu^{\prime}=0}^{\ell} \frac{\Gamma\left(\ell+3 / 2-\nu^{\prime}\right)}{\Gamma(3 / 2)} \frac{v^{\ell-\nu^{\prime}}}{\left(\ell-\nu^{\prime}\right) !} k_{2 \ell+1-2 \nu^{\prime}}\right) v^{\prime}=1 .
$$

Thus, we get the algebraic equation for the potential:

$$
\sum_{\nu^{\prime}=0}^{\ell} \frac{\Gamma\left(\ell+3 / 2-\nu^{\prime}\right)}{\Gamma(3 / 2)} \frac{\nu^{\ell+1-\nu^{\prime}}}{\left(\ell+1-\nu^{\prime}\right) !} k_{2 \ell+1-2 \nu^{\prime}}=x+c_{n} .
$$

\subsection{Superintegrable Hamiltonians and integrals of motion}

In this section we will discuss different ways to construct superintegrable Hamiltonians from the previous results.

(a) Superintegrable Hamiltonians by adding two Heisenberg Hamiltonians

Once we have the Heisenberg algebras $\left\langle H_{n x}, B_{n x}, 1\right\rangle,\left\langle H_{n y}, B_{n y}, 1\right\rangle$ we can write the superintegrable Hamiltonian

$$
H_{n}=H_{n x}+H_{n y}, \quad v_{n n}(x, y)=v_{n x}(x)+v_{n y}(y),
$$

whose 'extra' constant of motion of odd degree $n=2 \ell+1$ is given by 


$$
B_{n}=B_{n x}-B_{n y} .
$$

Remark that, in general these systems are only defined in a region of the plane $\mathbb{R}^{2}$. Some examples are:

(i) $v_{11}(x, y)=\alpha x+\beta y$.

(ii) $v_{33}(x, y)=\alpha \sqrt{x}+\beta \sqrt{y}, \quad x \geqslant 0, y \geqslant 0$.

(iii) $v_{55}(x, y)=\alpha x^{1 / 3}+\beta y^{1 / 3}$.

(iv) $v_{77}(x, y)=\alpha x^{1 / 4}+\beta y^{1 / 4}, \quad x \geqslant 0, y \geqslant 0$.

Example (ii) was considered in [8] as case 5.

In fact, we can build superintegrable systems by combining Heisenberg algebras of different orders,

$$
H_{m n}=H_{m x}+H_{n y}, \quad v_{m n}(x, y)=v_{m x}(x)+v_{n y}(y),
$$

whose 'extra' constant of motion of odd degree $\max (m=2 k+1, n=2 \ell+1)$ will be given by

$$
B_{2 k+1,2 \ell+1}=B_{2 k+1 x}-B_{2 \ell+1 y} .
$$

The corresponding potentials include linear combinations of different roots,

$$
v_{2 k+1,2 \ell+1}(x, y)=\alpha x^{1 /(k+1)}+\beta y^{1 /(\ell+1)} .
$$

Some special cases are:

(v) $v_{13}(x, y)=\alpha x+\beta \sqrt{y}, \quad y \geqslant 0$.

(vi) $v_{35}(x, y)=\alpha \sqrt{x}+\beta y^{1 / 3}, \quad x \geqslant 0$.

(vii) $v_{15}(x, y)=\alpha x+\beta y^{1 / 3}$.

Example (v) was included in [8] as case 7. Notice that since each one dimensional potential $v_{m x}(x)$ or $v_{n y}(y)$ is a monotonous function, these potentials $v_{n m}$ will not allow for any bounded motion.

(b) Global and local Hamiltonians in $\mathbb{R}^{2}$

As it is clear from example (ii) given above, some of the superintegrable Hamiltonians have potentials $v_{m n}(x, y)$ defined only in a region $\mathcal{D}$ of the plane. We may try to extend this Hamiltonian to the whole plane by pasting it with other superintegrable systems with potentials $v_{m n}^{i}(x, y)$ defined in disjoint (except for their boundaries) regions $\mathcal{D}^{i}$, such that they cover the whole plane, $\cup_{i} \mathcal{D}^{i}=\mathbb{R}^{2}$. For instance, considering the particular case $\alpha=\beta=1$, we can 'complete' the potential of case (ii) as follows:

$\tilde{v}_{33}(x, y)=\sqrt{|x|}+\sqrt{|y|}= \begin{cases}v_{33}^{1}=\sqrt{x}+\sqrt{y}, & \mathcal{D}^{1}=\{(x, y), x \geqslant 0, y \geqslant 0\} \\ v_{33}^{2}=\sqrt{-x}+\sqrt{y}, & \mathcal{D}^{2}=\{(x, y), x \leqslant 0, y \geqslant 0\} \\ v_{33}^{3}=\sqrt{-x}+\sqrt{-y}, & \mathcal{D}^{3}=\{(x, y), x \leqslant 0, y \leqslant 0\} \\ v_{33}^{4}=\sqrt{x}+\sqrt{-y}, & \mathcal{D}^{4}=\{(x, y), x \geqslant 0, y \leqslant 0\}\end{cases}$

Another example extended from case (i) is given by

$$
\tilde{v}_{11}=|x|+|y| \text {. }
$$

We can apply this 'pasting process' in order to produce a global potential such that it will allow for bounded trajectories, as it is the case of the extensions (2.47) or (2.48), given above. However, in these cases, the resulting system with global potential $\tilde{v}_{33}(x, y)$ will not be superintegrable since there is not a 'global' constant of motion for $\tilde{v}$. In a motion under $\tilde{v}_{33}(x, y)$, 
when the particle is in $\mathcal{D}^{1}$ the constant of motion is $B^{1}$, but when the particle crosses from the domain $\mathcal{D}^{1}$ to $\mathcal{D}^{2}$, the constant of motion will change and it will take a different value $B^{2}$, and so on. In this way after the particle has been $n_{1}$ times in the region $\mathcal{D}^{1}$, the constant of motion $B^{1}$ will have taken, in general, $n_{1}$ different values. Therefore, in general the motion will not be periodic. In figure 1 it is shown the plot of the 'pasted potential' given by (2.47). The motion of a particle in this potential is a superposition of two one-dimensional motions corresponding to the potentials $\tilde{v}_{x}=\sqrt{|x|}$ and $\tilde{v}_{y}=\sqrt{|y|}$. In this case, the ratio of the frequencies $\nu_{x}$ and $\nu_{y}$ is obtained by action-angle variables method and it is given by

$$
\frac{\nu_{x}}{\nu_{y}}=\left(\frac{E_{x}}{E_{y}}\right)^{3 / 2} \text {. }
$$

Only when this ratio is a rational number, the bounded motion in the plane will be periodic. Examples of periodic and non-periodic orbits for this pasted potential are given in figure 2. For 'global' superintegrable systems all the bounded motions are periodic and the trajectories look like deformed Lissajous curves $[10,11]$. We should remark that such trajectories are smooth and they do not present 'angles' when crossing the boundary of a domain. This point was not clearly explained in previous references $[7,8]$.

\section{(c) Superintegrable Hamiltonians in higher dimensions}

We can define superintegrable Hamiltonians in three or more dimensions. For instance, we simply add three one dimensional Heisenberg Hamiltonians to get a three dimensional Hamiltonian

$$
H_{n m p}=H_{n x}(x)+H_{m y}(y)+H_{p z}(z) .
$$

This new Hamiltonian is superintegrable. First of all, as it is separable, we have three constants of motion in involution, for instance: $H_{n x}(x), H_{m y}(y)$ and $H_{p z}(z)$. Second, as each Hamiltonian is of Heisenberg type, we can construct two additional independent constants of motion:

$$
B_{1}=B_{n x}-B_{m y}, \quad B_{2}=B_{m y}-B_{p z}
$$

The symmetry algebra of all the independent constants of motion is very easy to compute, it is essentially a subalgebra of the direct sum of one-dimensional Heisenberg algebras.

\section{Heisenberg-type higher order symmetries: the quantum problem}

In the framework of quantum mechanics, let us consider now a Hamiltonian operator $\mathcal{H}$ in one Cartesian coordinate $(2 m=1)$ :

$$
\mathcal{H}=P^{2}+V(X),
$$

where $P$ and $X$ are the momentum and position operators, satisfying the well known commutation relation

$$
[X, P]=\mathrm{i} \hbar .
$$

In the sequel, we will work in the coordinate representation of wave functions where the momentum operator is $P=-\mathrm{i} \hbar \mathrm{d} / \mathrm{d} x$ and the position operator $X$ is just the multiplication by the variable $x$.

Now, we introduce the notion of Heisenberg operator $\mathcal{B}_{n}$ for this Hamiltonian $\mathcal{H}$ as a $n$-order polynomial in the momentum operator $P$ with $x$-dependent coefficients, where the powers of the operator $P$ are placed 'on the right', that is: 


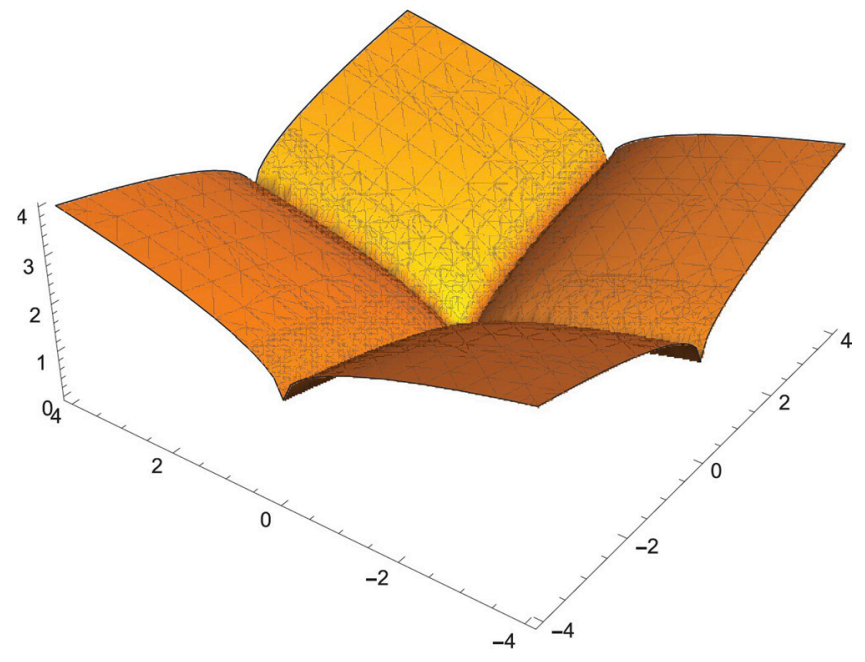

Figure 1. Plot of the potential $\tilde{v}_{33}=\sqrt{|x|}+\sqrt{|y|}$.
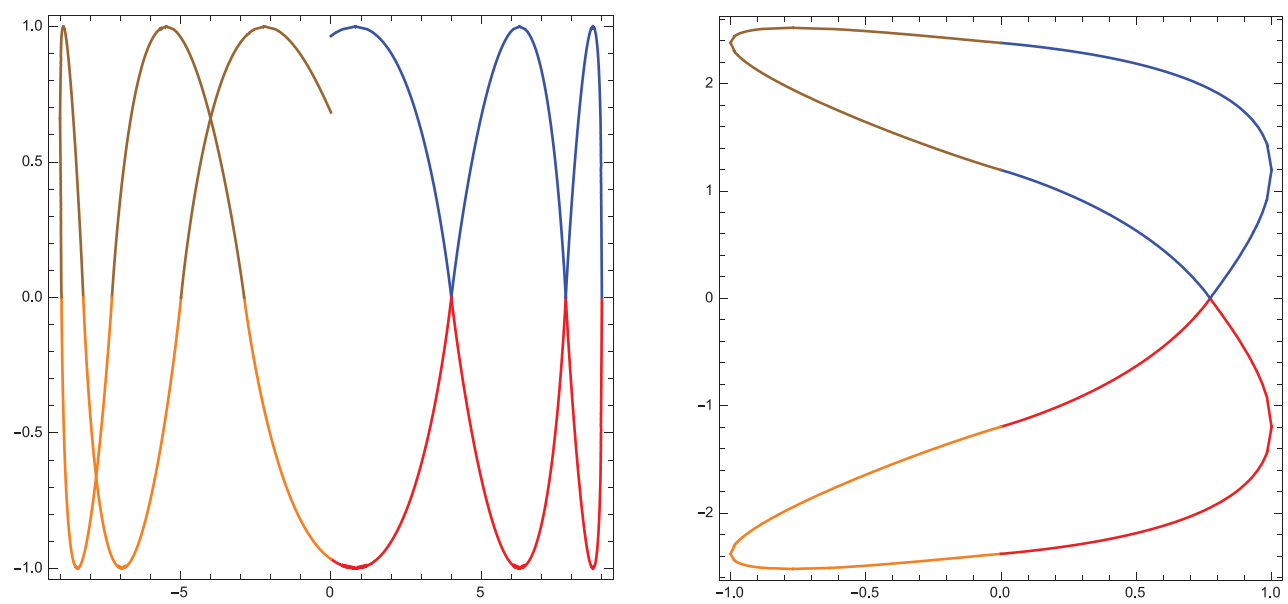

Figure 2. Plot of a non periodic trajectory corresponding to parameters $E_{x}=3, E_{y}=1, B=5$ (left) and a closed periodic trajectory with parameters $E_{x}=1, E_{y}=\sqrt[3]{4}, B=1$ (right). The pieces of trajectory corresponding to each quadrant of the plane are in a different color.

$$
\mathcal{B}_{n}=\sum_{k=0}^{n} F_{k}(x) P^{k}
$$

being $F_{k}(x)$ functions of the real variable $x$, to be determined. The Heisenberg function (3.3) must satisfy the following commutation relation with the Hamiltonian (3.1)

$$
\left[\mathcal{H}, \mathcal{B}_{n}\right]=\mathrm{i} \hbar \text {. }
$$


This condition eventually will gives us a condition on the potential, that will depend on $x$, but also on $n$; due to this fact, instead of (3.1) we will use the following notation for the Hamiltonian

$$
\mathcal{H}_{n}=P^{2}+V_{n}(x)
$$

Remark that if we take the formal adjoint of relation (3.4) we get

$$
\left[\mathcal{H}, \mathcal{B}_{n}^{\dagger}\right]=\mathrm{i} \hbar
$$

where $\mathcal{B}_{n}^{\dagger}$ is the adjoint differential operator of $\mathcal{B}_{n}$. Therefore,

$$
\left[\mathcal{H}, \mathcal{B}_{n}^{s}\right]=\mathrm{i} \hbar, \quad \mathcal{B}_{n}^{s}=\frac{1}{2}\left(\mathcal{B}_{n}+\mathcal{B}_{n}^{\dagger}\right)
$$

Hence, we can always assume the operator $\mathcal{B}_{n}$ in (3.4) to be a Hermitian differential operator.

In the following, for the sake of simplicity we will omit the explicit dependence of the functions $F_{\ell}(x)$ and $V_{\ell}(x)$ on the variable $x$.

\subsection{The potentials and the Heisenberg operators}

Now, we will find the form of the potentials and the operators $\mathcal{B}_{n}$ satisfying equation (3.4), for different values of $n$.

- Case $n=1$

The Heisenberg operator has the form

$$
\mathcal{B}_{1}=F_{0}+F_{1} P
$$

similar to its classical equivalent (2.3). Substituting (3.8) in (3.4), with $P=-\mathrm{i} \hbar \mathrm{d} / \mathrm{d} x$, we get the following system of ordinary differential equations

$$
F_{1}^{\prime}=0, \quad F_{0}^{\prime}=0, \quad F_{1} V_{1}^{\prime}=1 .
$$

where the primes denotes the derivative with respect to the variable $x$. Observe that this system is exactly the same obtained in the classical situation (2.5), without any 'quantum corrections'. Therefore, it is trivially solved and we get the same solutions as in the classical case:

$$
F_{1}=k_{1}, \quad F_{0}=k_{0}, \quad V_{1}(x)=\frac{x}{k_{1}}, \quad \mathcal{H}_{1}=P^{2}+\frac{x}{k_{1}}, \quad \mathcal{B}_{1}=k_{0}+k_{1} P,
$$

where $k_{1}$ and $k_{0}$ are integration constants; we have omitted a third irrelevant additive integration constant in the potential.

- Case $n=2$

The Heisenberg operator takes the form

$$
\mathcal{B}_{2}=F_{0}+F_{1} P+F_{2} P^{2} .
$$

Substituting (3.11) in (3.4) we get the following set of equations:

$$
F_{2}^{\prime}=0, \quad F_{1}^{\prime}=0, \quad F_{0}^{\prime}=F_{2} V_{2}^{\prime}, \quad 1=F_{1} V_{2}^{\prime}+\mathrm{i} \hbar\left(F_{0}^{\prime \prime}-F_{2} V_{2}^{\prime \prime}\right) .
$$

Here we have a quantum version of the classical result (2.10), with a quantum correction of first order in $\hbar$. Nevertheless, this $\hbar$ dependence is only apparent because the coefficient of $\hbar$ turns out to be zero. In fact, the solution of this system is: 


$$
F_{2}=k_{2}, \quad F_{1}=k_{1}, \quad F_{0}=k_{0}+k_{2} V_{2},
$$

where $k_{0}, k_{1}$ and $k_{2}$ are integration constants ( $k_{0}$ is irrelevant and will be neglected), and the potential is exactly the same obtained in the case $n=1$ given in equation (3.10):

$$
V_{2}(x)=\frac{x}{k_{1}}=V_{1}(x), \quad \mathcal{H}_{2}=P^{2}+\frac{x}{k_{1}} .
$$

Hence, the operator $\mathcal{B}_{2}$ has the expression:

$$
\mathcal{B}_{2}=k_{1} P+k_{2}\left(P^{2}+V_{2}\right)=B_{1}+k_{2} H_{2} .
$$

The term $k_{2} H_{2}$ is redundant, and therefore the nontrivial solution reduces to $k_{1} P$. Hence, the case $n=2$ does not provide new interesting results, as its solution coincides exactly with the one obtained in case $n=1$.

- Case $n=3$

Now, the Heisenberg operator is

$$
\mathcal{B}_{3}=F_{0}+F_{1} P+F_{2} P^{2}+F_{3} P^{3} .
$$

Substituting (3.16) in (3.4) we get the following set of equations:

$$
\begin{aligned}
2 F_{3}^{\prime} & =0, \\
2 F_{2}^{\prime} & =0, \\
2 F_{1}^{\prime} & =3 F_{3} V_{3}^{\prime}, \\
2 F_{0}^{\prime} & =2 F_{2} V_{3}^{\prime}+\mathrm{i} \hbar\left(F_{1}^{\prime \prime}-3 F_{3} V_{3}^{\prime \prime}\right), \\
1 & =F_{1} V_{3}^{\prime}+\mathrm{i} \hbar\left(F_{0}^{\prime \prime}-F_{2} V_{3}^{\prime \prime}\right)-\hbar^{2} F_{3} V_{3}^{\prime \prime \prime} .
\end{aligned}
$$

Observe the presence on the right hand side of this system of some nontrivial quantum corrections which obviously were not present in the classical case (2.14): nonvanishing terms containing powers of $\hbar$, that eventually will make more difficult to find the solution. Indeed, the 'quantum system' does not have the separation property between the functions with even indices $\left(F_{0}, F_{2}, \ldots\right)$ and odd indices $\left(F_{1}, F_{3}, \ldots\right)$, something that is true in the classical case, as can be clearly seen in (2.36).

The solution of the system (3.17) exhibits the explicit presence of quantum terms:

$$
F_{3}=k_{3}, \quad F_{2}=k_{2}, \quad F_{1}=k_{1}+\frac{3}{2} k_{3} V_{3}, \quad F_{0}=k_{0}+k_{2} V_{3}-\frac{3}{4} \mathrm{i} \hbar k_{3} V_{3}^{\prime},
$$

where the $k_{\ell}$ are integration constants ( $k_{0}$ will be omitted in the sequel), and the potential $V_{3}(x)$ must satisfy the following third order nonlinear differential equation

$$
k_{3}\left(\hbar^{2} V_{3}^{\prime \prime \prime}-6 V_{3} V_{3}^{\prime}\right)-4 k_{1} V_{3}^{\prime}+4=0,
$$

which is the 'quantum version' of (2.15): indeed, if we make $\hbar=0$ in (3.19) we recover (2.15).

The nonlinear differential equation (3.19) can be integrated once:

$$
k_{3}\left(\hbar^{2} V_{3}^{\prime \prime}-3 V_{3}^{2}\right)-4 k_{1} V_{3}+4 x+c_{3}=0 .
$$

Remark that the information really new in the case $n=3$ comes from the term with coefficient $k_{3}$ : indeed, if for example we put $k_{3}=0$ in (3.20) we go back to the result (3.14). If we take $k_{1}=0$ we have

$$
k_{3}\left(\hbar^{2} V_{3}^{\prime \prime}-3 V_{3}^{2}\right)+4 x+c_{3}=0
$$


that can be finally reduced to the first Painlevé equation

$$
\frac{\mathrm{d}^{2} W_{3}}{\mathrm{~d} z^{2}}=6 W_{3}^{2}+z
$$

by means of the following transformations:

$$
x=-\operatorname{sign}\left(k_{3}\right) \sqrt[5]{\frac{\left|k_{3}\right| \hbar^{4}}{2}} z-\frac{c_{3}}{4}, \quad V_{3}=2\left(\frac{2 \hbar}{\left|k_{3}\right|}\right)^{2 / 5} W_{3} .
$$

Notice that if we take $\hbar=0$ in (3.20), the equation reduces to the classical solution obtained previously in (2.18), without necessity of any further integration. Obviously in the quantum case the presence of the terms with powers of $\hbar$ make the whole story more interesting and also more difficult to deal with.

The operator $\mathcal{B}_{3}$ can be written as

$$
\mathcal{B}_{3}=\mathcal{B}_{1}+k_{2} \mathcal{H}_{3}+k_{3} \mathcal{B}_{3}^{0},
$$

and, obviously, only the $\mathcal{B}_{3}^{0}$ term gives us new interesting information:

$\mathcal{B}_{3}^{0}=P^{3}+\frac{3}{2} V_{3} P-\frac{3}{4} \mathrm{i} \hbar V_{3}^{\prime}=P^{3}+\frac{3}{2} \frac{V_{3} P+P V_{3}}{2}=-\frac{1}{2}\left(P^{3}-3 \frac{\mathcal{H}_{3} P+P \mathcal{H}_{3}}{2}\right)$.

We see that $\mathcal{B}_{3}^{0}$ is the symmetrized version of the classical expression (2.19).

- Case $n=4$

The integral of motion is now of fourth order. The problem is solved as in the previous cases and we obtain that the potential $V_{4}(x)$ must satisfy a third order nonlinear differential equation which is exactly the same as (3.19) for $V_{3}(x)$, therefore $V_{4}(x)=V_{3}(x)$. The integral of motion $\mathcal{B}_{4}$ has the form:

$$
\mathcal{B}_{4}=\mathcal{B}_{1}+k_{2} \mathcal{H}_{4}+k_{3} \mathcal{B}_{3}^{0}+k_{4}\left(\mathcal{H}_{4}\right)^{2} .
$$

There is no new interesting information, because the terms containing powers of $\mathcal{H}_{4}$ are irrelevant, and the other two components, $\mathcal{B}_{1}$ and $\mathcal{B}_{3}^{0}$ have been already obtained. A similar situation appeared in case $n=2$, and is typical of all the even cases.

- Case $n=5$

The integral of motion is

$$
\mathcal{B}_{5}=F_{0}+F_{1} P+F_{2} P^{2}+F_{3} P^{3}+F_{4} P^{4}+F_{5} P^{5} .
$$

Substituting (3.27) in (3.4), we get the following set of equations:

$$
\begin{aligned}
2 F_{5}^{\prime} & =0, \\
2 F_{4} & =0, \\
2 F_{3}^{\prime} & =5 F_{5} V_{5}^{\prime}, \\
2 F_{2}^{\prime} & =4 F_{4} V_{5}^{\prime}+\mathrm{i} \hbar\left(F_{3}^{\prime \prime}-10 F_{5} V_{5}^{\prime \prime}\right), \\
2 F_{1}^{\prime} & =3 F_{3} V_{5}^{\prime}+\mathrm{i} \hbar\left(F_{2}^{\prime \prime}-6 F_{4} V_{5}^{\prime \prime}\right)-10 \hbar^{2} F_{5} V_{5}^{\prime \prime \prime}, \\
2 F_{0}^{\prime} & =2 F_{2} V_{5}^{\prime}+\mathrm{i} \hbar\left(F_{1}^{\prime \prime}-3 F_{3} V_{5}^{\prime \prime}\right)-4 \hbar^{2} F_{4} V_{5}^{\prime \prime \prime}+5 \mathrm{i} \hbar^{3} F_{5} V_{5}^{(i v)}, \\
1 & =F_{1} V_{5}^{\prime}+\mathrm{i} \hbar\left(F_{0}^{\prime \prime}-F_{2} V_{5}^{\prime \prime}\right)-\hbar^{2} F_{3} V_{5}^{\prime \prime \prime}+\mathrm{i} \hbar^{3} F_{4} V_{5}^{(i v)}+\hbar^{4} F_{5} V_{5}^{(v)} .
\end{aligned}
$$

Observe that this quantum version of the classical result (2.22) has quantum corrections up to order $\hbar^{4}$. Looking at (3.28) it is quite obvious that the quantum corrections are 
growing in importance. In spite of the imposing aspect of this system, it is possible to find the explicit solution of the functions $F_{\ell}$ appearing there:

$$
\begin{aligned}
& F_{5}=k_{5}, \quad F_{4}=k_{4}, \quad F_{3}=k_{3}+\frac{5}{2} k_{5} V_{5}, \\
& F_{2}=k_{2}+2 k_{4} V_{5}-\frac{15}{4} \mathrm{i} \hbar k_{5} V_{5}^{\prime}, \\
& F_{1}=k_{1}+\frac{3}{2} k_{3} V_{5}-2 \mathrm{i} \hbar k_{4} V_{5}^{\prime}+k_{5}\left(\frac{15}{8} V_{5}^{2}-\frac{25}{8} \hbar^{2} V_{5}^{\prime \prime}\right), \\
& F_{0}=k_{0}+k_{2} V_{5}-\frac{6}{8} \mathrm{i} \hbar k_{3} V_{5}^{\prime}+k_{4}\left(V_{5}^{2}-\hbar^{2} V_{5}^{\prime \prime}\right)-\frac{15}{16} \mathrm{i} \hbar k_{5}\left(2 V_{5} V_{5}^{\prime}+(\mathrm{i} \hbar)^{2} V_{5}^{\prime \prime \prime}\right),
\end{aligned}
$$

where the $k_{\ell}$ are integration constants. The potential $V_{5}(x)$ must satisfy the following fifth order nonlinear differential equation

$$
k_{5}\left(\hbar^{4} V_{5}^{(v)}+30 V_{5}^{2} V_{5}^{\prime}-20 \hbar^{2} V_{5}^{\prime} V_{5}^{\prime \prime}-10 \hbar^{2} V_{5} V_{5}^{\prime \prime \prime}\right)+k_{3}\left(24 V_{5} V_{5}^{\prime}-4 \hbar^{2} V_{5}^{\prime \prime \prime}\right)+16 k_{1} V_{5}^{\prime}=16
$$

Some remarks are in order here: (i) if we take $\hbar=0$ in this nonlinear fifth-order differential equation, we recover the simple first order differential equation (2.23) of the classical $n=5$ case; (ii) if we take $k_{5}=0$ we go back to the case $n=3$ studied before (3.19); (iii) only constants $k_{\ell}$ with odd indices are present in (3.29): the constants $k_{0}, k_{2}$ and $k_{4}$ does not play any role in the solution of the problem we are studying (in particular, $k_{0}$ will be neglected in the sequel); (iv) the equation can be integrated once to give

$$
k_{5}\left(\hbar^{4} V_{5}^{(i v)}+10 V_{5}^{3}-5 \hbar^{2}\left(2 V_{5} V_{5}^{\prime \prime}+\left(V_{5}^{\prime}\right)^{2}\right)\right)+k_{3}\left(12\left(V_{5}\right)^{2}-4 \hbar^{2} V_{5}^{\prime \prime}\right)+16 k_{1} V_{5}=16 x+c_{5} .
$$

As in the case $n=3$, the new relevant information of the present case is obtained by taking $k_{1}=k_{3}=0$ in $(3.30)$

$$
\hbar^{4} V_{5}^{(i v)}-10 \hbar^{2}\left(V_{5} V_{5}^{\prime \prime}+\frac{1}{2}\left(V_{5}^{\prime}\right)^{2}\right)+10 V_{5}^{3}=\frac{16 x+c_{5}}{k_{5}} .
$$

Remark that if $\hbar=0$, we recover basically the classical $n=5$ result (2.25). With the simple transformations

$$
V_{5}=2 \hbar^{2} W_{5}, \quad x=z-\frac{c_{5}}{16}, \quad \kappa=\frac{8}{\hbar^{6} k_{5}},
$$

equation (3.31) turns out to be

$$
\frac{\mathrm{d}^{4} W_{5}}{\mathrm{~d} z^{4}}=20 W_{5} \frac{\mathrm{d}^{2} W_{5}}{\mathrm{~d} z^{2}}+10\left(\frac{\mathrm{d} W_{5}}{\mathrm{~d} z}\right)^{2}-40 W_{5}^{3}+\kappa z,
$$

which appears in the list of fourth order Painléve equations of polynomial class, classified by Cosgrove [12]: it is precisely the so-called equation F-V (see equation (2.67) of [12] with $\alpha=\beta=0$ ). Equation F-V has the Painléve property and arises as group-invariant reduction of the KdV5 equation (a member of the KdV hierarchy). It is also a member of the so-called Painléve-I hierarchy and is denoted by the notation ${ }_{1} P_{4}$ [13]. It is conjectured that $\mathrm{F}-\mathrm{V}$ (in the nonautonomous case) defines a new transcendent in the sense that the general solution of F-V cannot be expressed in terms of known transcendents including the six Painléve transcendents, elliptic, hyperelliptic, abelian and automorphic functions. 
The second order Painléve transcendents $P_{I}, P_{I I}, P_{I V}$ as quantum potentials have appeared previously $[4,7,8,14,15]$. A fourth order form of the potential equation which can be integrated in terms of solutions of the fourth Painléve equation $P_{I V}$ first appeared in [4]. To the best of our knowledge, the occurrence of a genuine fourth order Painléve transcendent as potential is new. The surprising connection of superintegrability in the quantum case with soliton theory of infinite-dimensional integrable nonlinear systems manifests itself here once again.

The Heisenberg operator $\mathcal{B}_{5}$ has the form:

$$
\mathcal{B}_{5}=\mathcal{B}_{1}+k_{2} \mathcal{H}_{5}+k_{3} \mathcal{B}_{3}^{0}+k_{4} \mathcal{H}_{5}^{2}+k_{5} \mathcal{B}_{5}^{0}
$$

where, the essentially new term is given by

$$
\begin{aligned}
\mathcal{B}_{5}^{0} & =P^{5}+\frac{5}{2} V_{5} P^{3}-\frac{15}{4} \mathrm{i} \hbar V_{5}^{\prime} P^{2}+\frac{5}{8}\left(3 V_{5}^{2}-5 \hbar^{2} V_{5}^{\prime \prime} P-\frac{15}{16} \mathrm{i} \hbar\left(2 V_{5} V_{5}^{\prime}-\hbar^{2} V_{5}^{\prime \prime \prime}\right)\right. \\
& =\frac{3}{8}\left(P^{5}-\frac{5}{3}\left(P H P^{2}+P^{2} H P\right)+\frac{5}{2}\left(H^{2} P+P H^{2}\right)\right) .
\end{aligned}
$$

Notice that the last expression is a symmetrized version of the corresponding classical function (2.27).

- Case $n=6$

As we have already seen in the previous analysis, the even cases do not provide new information, and therefore we will skip the case $n=6$.

- $n=7$

This is the last case that we will consider in this paper. The Heisenberg operator is of seventh order,

$$
\mathcal{B}_{7}=F_{0}+F_{1} P+F_{2} P^{2}+F_{3} P^{3}+F_{4} P^{4}+F_{5} P^{5}+F_{6} P^{6}+F_{7} P^{7} .
$$

Substituting (3.36) in (3.4) we get the following set of equations:

$$
\begin{aligned}
2 F_{7}^{\prime} & =0, \\
2 F_{6}^{\prime} & =0, \\
2 F_{5}^{\prime} & =7 F_{7} V_{7}^{\prime}, \\
2 F_{4}^{\prime} & =6 F_{6} V_{7}^{\prime}+\mathrm{i} \hbar\left(F_{5}^{\prime \prime}-21 F_{7} V_{7}^{\prime \prime}\right), \\
2 F_{3}^{\prime} & =5 F_{5} V_{7}^{\prime}+\mathrm{i} \hbar\left(F_{4}^{\prime \prime}-15 F_{6} V_{7}^{\prime \prime}\right)-35 \hbar^{2} F_{7} V_{7}^{\prime \prime \prime}, \\
2 F_{2}^{\prime} & =4 F_{4} V_{7}^{\prime}+\mathrm{i} \hbar\left(F_{3}^{\prime \prime}-10 F_{5} V_{7}^{\prime \prime}\right)-20 \hbar^{2} F_{6} V_{7}^{\prime \prime \prime}+35 \mathrm{i} \hbar^{3} F_{7} V_{7}^{(i v)}, \\
2 F_{1}^{\prime} & =3 F_{3} V_{7}^{\prime}+\mathrm{i} \hbar\left(F_{2}^{\prime \prime}-6 F_{4} V_{7}^{\prime \prime}\right)-10 \hbar^{2} F_{5} V_{7}^{\prime \prime \prime}+15 \mathrm{i} \hbar^{3} F_{6} V_{7}^{(i v)}+21 \hbar^{4} F_{7} V_{7}^{(v)}, \\
2 F_{0}^{\prime} & =2 F_{2} V_{7}^{\prime}+\mathrm{i} \hbar\left(F_{1}^{\prime \prime}-3 F_{3} V_{7}^{\prime \prime}\right)-4 \hbar^{2} F_{4} V_{7}^{\prime \prime \prime}+5 \mathrm{i} \hbar^{3} F_{5} V_{7}^{(i v)}+6 \hbar^{4} F_{6} V_{7}^{(v)}-7 \mathrm{i} \hbar^{5} F_{7} V_{7}^{(v i)}, \\
1 & =F_{1} V_{7}^{\prime}+\mathrm{i} \hbar\left(F_{0}^{\prime \prime}-F_{2} V_{7}^{\prime \prime}\right)-\hbar^{2} F_{3} V_{7}^{\prime \prime \prime}+\mathrm{i} \hbar^{3} F_{4} V_{7}^{(i v)}+\hbar^{4} F_{5} V_{7}^{(v)}-\mathrm{i} \hbar^{5} F_{6} V_{7}^{(v i)}-\hbar^{6} F_{7} V_{7}^{(v i i)} .
\end{aligned}
$$

The solution of this system is: 


$$
\begin{aligned}
F_{7}= & k_{7}, \quad F_{6}=k_{6}, \quad F_{5}=k_{5}+\frac{7}{2} k_{7} V_{7}, \quad F_{4}=k_{4}+3 k_{6} V_{7}-\frac{35}{4} \mathrm{i} \hbar k_{7} V_{7}^{\prime}, \\
F_{3}= & k_{3}+\frac{5}{2} k_{5} V_{7}-6 \mathrm{i} \hbar k_{6} V_{7}^{\prime}+\frac{35}{8} k_{7}\left(V_{7}^{2}-3 \hbar^{2} V_{7}^{\prime \prime}\right) \\
F_{2}= & k_{2}+2 k_{4} V_{7}-\frac{15}{4} \mathrm{i} \hbar k_{5} V_{7}^{\prime}+k_{6}\left(3 V_{7}^{2}-7 \hbar^{2} V_{7}^{\prime \prime}\right)-\frac{35}{16} \mathrm{i} \hbar k_{7}\left(6 V_{7} V_{7}^{\prime}+5(\mathrm{i} \hbar)^{2} V_{7}^{\prime \prime \prime}\right), \\
F_{1}= & k_{1}+\frac{3}{2} k_{3} V_{7}-2 i k_{4} \hbar V_{7}^{\prime}+\frac{5}{8} k_{5}\left(3 V_{7}^{2}-5 \hbar^{2} V_{7}^{\prime \prime}\right)+\mathrm{i} \hbar k_{6}\left(4 \hbar^{2} V_{7}^{\prime \prime \prime}-6 V_{7} V_{7}^{\prime}\right) \\
& +\frac{1}{32} k_{7}\left(161 \hbar^{4} V_{7}^{(i v)}-350 \hbar^{2} V_{7} V_{7}^{\prime \prime}-245 \hbar^{2}\left(V_{7}^{\prime}\right)^{2}+70 V_{7}^{3}\right), \\
F_{0}= & k_{0}+k_{2} V_{7}-\frac{3}{4} i k_{3} \hbar V_{7}^{\prime}+k_{4}\left(V_{7}^{2}-\hbar^{2} V_{7}^{\prime \prime}\right)+\frac{15}{16} \mathrm{i} \hbar k_{5}\left(\hbar^{2} V_{7}^{\prime \prime \prime}-2 V_{7} V_{7}^{\prime}\right) \\
& +k_{6}\left(\hbar^{4} V_{7}^{(i v)}-3 \hbar^{2} V_{7} V_{7}^{\prime \prime}-2 \hbar^{2}\left(V_{7}^{\prime}\right)^{2}+V_{7}^{3}\right) \\
& +\frac{21}{64} \mathrm{i} \hbar k_{7}\left(-3 \hbar^{4} V_{7}^{(v)}+10 \hbar^{2} V_{7} V_{7}^{\prime \prime \prime}-10 V_{7}^{2} V_{7}^{\prime}+20 \hbar^{2} V_{7}^{\prime} V_{7}^{\prime \prime}\right),
\end{aligned}
$$

where the $k_{\ell}$ are integration constants, and the potential $V_{7}$ must satisfy the following seventh order nonlinear differential equation:

$$
\begin{aligned}
k_{7} & {\left[\hbar^{6} V_{7}^{(v i i)}-14 \hbar^{4}\left(V_{7} V_{7}^{(v)}+3 V_{7}^{(i v)} V_{7}^{\prime}+5 V_{7}^{\prime \prime \prime} V_{7}^{\prime \prime}\right)+70 \hbar^{2}\left(V_{7}^{2} V_{7}^{\prime \prime \prime}+\left(V_{7}^{\prime}\right)^{3}+4 V_{7} V_{7}^{\prime} V_{7}^{\prime \prime}\right)\right.} \\
& \left.-140 V_{7}^{3} V_{7}^{\prime}\right]+4 k_{5}\left[-\hbar^{4} V_{7}^{(v)}+10 \hbar^{2}\left(V_{7} V_{7}^{\prime \prime \prime}+2 V_{7}^{\prime} V_{7}^{\prime \prime}\right)-30 V_{7}^{2} V_{7}^{\prime}\right] \\
& +16 k_{3}\left[\hbar^{2} V_{7}^{\prime \prime \prime}-6 V_{7} V_{7}^{\prime}\right]-64 k_{1} V_{7}^{\prime}+64=0 .
\end{aligned}
$$

Remark that only $k_{\ell}$ with odd indices are present: the constants $k_{0}, k_{2}, k_{4}$ and $k_{6}$ do not play any role in the solution of the problem we are studying (indeed, as in the previous cases, we will omit $k_{0}$ from now on). If we consider the limit case $\hbar=0$, the nonlinear differential equation (3.38) reduces to the much simpler equation (2.30). In spite of its formidable aspect, equation (3.38) can be integrated once:

$$
\begin{aligned}
k_{7} & {\left[-\hbar^{6} V_{7}^{(v i)}+7 \hbar^{4}\left(2 V_{7} V_{7}^{(i v)}+4 V_{7}^{\prime \prime \prime} V_{7}^{\prime}+3\left(V_{7}^{\prime \prime}\right)^{2}\right)-70 \hbar^{2}\left(V_{7}^{2} V_{7}^{\prime \prime}+\left(V_{7}^{\prime}\right)^{2} V_{7}\right)+35 V_{7}^{4}\right] } \\
& +4 k_{5}\left[\hbar^{4} V_{7}^{(i v)}-5 \hbar^{2}\left(2 V_{7} V_{7}^{\prime \prime}+\left(V_{7}^{\prime}\right)^{2}\right)+10 V_{7}^{3}\right]-16 k_{3}\left[\hbar^{2} V_{7}^{\prime \prime}-3\left(V_{7}\right)^{2}\right]+64 k_{1} V_{7}=64 x+c_{7}
\end{aligned}
$$

If we consider here $\hbar=0$, all the terms with derivatives of $V_{7}(x)$ completely disappear, and the corresponding fourth order polynomial equation (2.31) is obtained for the classical potential.

The special case of (3.39) where $k_{1}=k_{3}=k_{5}=0$ and $k_{7} \neq 0$ gives rise to a novel potential $\hbar^{6} V_{7}^{(v i)}-7 \hbar^{4}\left(2 V_{7} V_{7}^{(i v)}+4 V_{7}^{\prime \prime \prime} V_{7}^{\prime}+3\left(V_{7}^{\prime \prime}\right)^{2}\right)+70 \hbar^{2}\left(V_{7}^{2} V_{7}^{\prime \prime}+\left(V_{7}^{\prime}\right)^{2} V_{7}\right)-35 V_{7}^{4}=\frac{64 x+c_{7}}{-k_{7}}$.

We have checked if equation (3.40) passes the Painlevé test, what is only a necessary condition for the equation to possess the Painlevé property. The resonances occur at $r=2,4,5,7,10$ at which all compatibility conditions are satisfied which implies that the test is passed. This feature is typical of all quantum potentials obtained so far. The simple transformations

$$
V_{7}(x)=\hbar^{2} W_{7}(x), \quad x=z-\frac{c_{7}}{64}, \quad \kappa=-\frac{64}{k_{7} \hbar^{8}},
$$


allows to transfer the dependence on $\hbar$ of the whole equation to the independent variable, transforming equation (3.40) into

$W_{7}^{(v i)}=14 W_{7} W_{7}^{(i v)}+28 W_{7}^{\prime \prime \prime} W_{7}^{\prime}+21\left(W_{7}^{\prime \prime}\right)^{2}-70 W_{7}^{2} V_{7}^{\prime \prime}-70\left(W_{7}^{\prime}\right)^{2} W_{7}+35 W_{7}^{4}+\kappa z$.

As we have already mentioned, this sixth-order nonlinear differential equation correspond to some sixth-order Painlevé equation to be determined.

The integral of motion $\mathcal{B}_{7}$ has the form:

$$
\mathcal{B}_{7}=k_{1} P+k_{2} \mathcal{H}_{7}+k_{3} \mathcal{B}_{3}^{0}+k_{4} \mathcal{H}_{7}^{2}+k_{5} \mathcal{B}_{5}^{0}+k_{6} \mathcal{H}_{7}^{3}+k_{7} \mathcal{B}_{7}^{0},
$$

where, again, new information comes only from the last term:

$$
\begin{aligned}
\mathcal{B}_{7}^{0}= & P^{7}+\frac{7}{2} V_{7} P^{5}-\frac{35}{4} \mathrm{i} \hbar V_{7}^{\prime} P^{4}+\frac{35}{8}\left(V_{7}^{2}-3 \hbar^{2} V_{7}^{\prime \prime}\right) P^{3}-\frac{35}{16} \mathrm{i} \hbar\left(6 V_{7} V_{7}^{\prime}-5 \hbar^{2} V_{7}^{\prime \prime \prime}\right) P^{2} \\
& +\frac{1}{32}\left(161 \hbar^{4} V_{7}^{(i v)}-350 \hbar^{2} V_{7} V_{7}^{\prime \prime}-245 \hbar^{2}\left(V_{7}^{\prime}\right)^{2}+70 V_{7}^{3}\right) P \\
& -\frac{21}{64} \mathrm{i} \hbar\left(3 \hbar^{4} V_{7}^{(v)}-10 \hbar^{2} V_{7} V_{7}^{\prime \prime \prime}+10 V_{7}^{2} V_{7}^{\prime}-20 \hbar^{2} V_{7}^{\prime} V_{7}^{\prime \prime}\right)
\end{aligned}
$$

\subsection{Superintegrable Hamiltonians and their symmetries}

Once we have the Heisenberg Hamiltonians $\left\langle\mathcal{H}_{m x}, \mathcal{B}_{m x}, 1\right\rangle$, and $\left\langle\mathcal{H}_{n y}, \mathcal{B}_{n y}, 1\right\rangle$ we can write a superintegrable Hamiltonian by adding them just as in the classical context:

$$
\mathcal{H}_{m n}=\mathcal{H}_{m x}+\mathcal{H}_{n y}, \quad V_{m n}(x, y)=V_{m x}(x)+V_{n y}(y),
$$

where the degrees $m$ and $n$ of each Hamiltonian can be different. The third symmetry of odd degree given by $\max (m, n)$ is

$$
\mathcal{B}_{m n}=\mathcal{B}_{m x}-\mathcal{B}_{n y}
$$

In this way we can trivially extend this method to get superintegrable Hamiltonians in three or higher dimensions.

However, the one dimensional potentials involved are given by solutions of nonlinear differential equations that are not well known in much detail. For instance, some of the potentials may have singularities or may not be well defined in the whole real line. This can depend on the initial conditions imposed to the solutions. In this sense the study of all the possible potentials is as complex as the classification of the solutions of such nonlinear equations.

In the quantum case the pasting of 'local' superintegrable potentials in order to get a potential defined in the whole plane is out of place. For instance, in the same way as (2.48), we could define the potential

$$
\tilde{V}_{11}=|x|+|y| \text {. }
$$

But we can not apply any property related to superintegrability to this two-dimensional system. Here, we can not act as in the classical case, pasting the trajectories of different domains. 


\section{Conclusions}

In this work we have carried out a systematic study of superintegrable Hamiltonian systems separable in Cartesian coordinates such that each component is of Heisenberg type. A onedimensional Hamiltonian $H_{n}$ is said to be of Heisenberg type in the classical context if there is a function $B_{n}$ of degree $n$ in the momentum variable such that the Heisenberg Poisson commutator $\left\{H_{n}, B_{n}\right\}=1$ is satisfied. In the quantum frame a similar definition applies for a Hamilton operator $\mathcal{H}_{n}$ and a polynomial operator $\mathcal{B}_{n}$ that satisfy the commutator $\left[\mathcal{H}_{n}, \mathcal{B}_{n}\right]=\mathrm{i} \hbar$.

In the classical case we have found a general solution to this problem for any value of $n$. The relevant solutions are realized for the odd values $n=2 \ell+1$. The potentials of this type of Hamiltonians satisfy an algebraic equation of degree $\ell+1$. A representative potential for such a value is given by a root of index $\ell+1: v_{2 \ell+1}(x) \propto x^{1 /(\ell+1)}, \ell \in \mathbb{N}$. Some of the resulting superintegrable Hamiltonians are defined in a region of the plane (in the case of two Cartesian coordinates) so that we are lead to a restricted concept of superintegrability. This type of potentials do not allow for classical bounded motions, so that a particle that initially is in one of these regions, in general after a time will leave it and cross to another region where the superintegrability is not satisfied. In conclusion, for some cases we can describe only a part of the motion by means of the superintegrability properties for such a kind of Heisenberg systems.

In the quantum case it is possible to work out the solutions for any value of $n$, but we have not found closed expressions. It is shown that, as in the classical case, the odd values $n=2 \ell+1$ are relevant. For some values of $\ell$, the expression for the operator $\mathcal{B}_{2 \ell+1}$ in terms of the potential function $V_{2 \ell+1}(x)$ has been explicitly computed, as well as the differential equation that $V_{2 \ell+1}(x)$ must satisfy. Contrary to the corresponding classical analog, here the equations (except for the case $\ell=0$ that can be integrated) are not algebraic, but nonlinear differential equations that can not be integrated in terms of known special functions. In fact, they belong to a type of higher order Painlevé equations, starting with Painlevé I for $\ell=1$. Given the equations for the potential and the expressions for the Heisenberg operators of the quantum problem in terms of the potential, then if we perform the classical limit $\hbar \rightarrow 0$, the corresponding classical equations for $v_{2 \ell+1}(x)$, as well as the classical expressions $B_{2 \ell+1}$ are recovered, in a certain sense.

Some particular solutions of the general approach contained in this paper can be found in previous references $[4,7,8]$. For example, in [4] the symmetries of the two-dimensional Euclidean systems separable in Cartesian coordinates, up to third order, are exhaustively studied; the results include as particular cases all our solutions up to order three: In the quantum systems these potentials are labeled as (Q.17) and (Q.20), while in the classical framework are the cases (C.5) and (C.7). Reference [7] analyses the same problem as [4] paying attention to the algebraic structure of the symmetries. Some of the potentials they obtained (cases 5, 7 and 8 , where the symmetry algebra is Heisenberg) are the same as in our work. We have carefully explained that the local superintegrability affect the trajectories corresponding to these three cases. Another reference dealing with a similar strategy is [5], where the author is also searching for higher order symmetries, for the same type of systems, by means of ladder operators. However, the difference is that we use as the basic ingredient the Heisenberg algebra instead of the ladder algebra.

In conclusion, we have shown here that a fruitful way to find higher order symmetries of classical and quantum systems can be based on the algebraic properties of the corresponding Hamiltonian. In the present work this key idea is successfully implemented by looking for superintegrable Hamiltonians of Heisenberg type. 


\section{Acknowledgments}

We acknowledge the financial support of the Spanish MINECO (Project MTM2014-57129C2-1-P) and Junta de Castilla y León (VA057U16). F Güngör and Ş Kuru acknowledge the warm hospitality during their stays at Department of Theoretical Physics, University of Valladolid, Spain.

\section{References}

[1] Miller W Jr, Post S and Winternitz P 2013 Classical and quantum superintegrability with applications J. Phys. A: Math. Theor. 46423001

[2] Fushchych W I and Nikitin A G 1997 Higher symmetries and exact solutions of linear and nonlinear Schrödinger equation J. Math. Phys. 385944

[3] Doebner H-D and Zhdanov R Z 1999 The stationary KdV hierarchy and so(2,1) as a spectrum generating algebra J. Math. Phys. 404995

[4] Gravel S 2004 Hamiltonians separable in Cartesian coordinates and third-order integrals of motion J. Math. Phys. 451003

[5] Marquette I 2011 An infinite family of superintegrable systems from higher order ladder operators and supersymmetry J. Phys. A: Conf. Ser. 284012047

[6] Post S and Winternitz P 2015 General $n$th order integrals of motion in the Euclidean plane J. Phys. A: Math. Theor. 48405201

[7] Marquette I and Winternitz P 2007 Polynomial Poisson algebras for classical superintegrable systems with a third-order integral of motion J. Math. Phys. 48012902

[8] Marquette I and Winternitz P 2008 Superintegrable systems with third order integrals of motion J. Phys. A: Math. Theor. 41304031

[9] Goldstein H, Poole CP and Safko J L 2001 Classical Mechanics 3rd edn (New York: Addison-Wesley)

[10] Calzada J A, Kuru Ş and Negro J 2008 Superintegrable Lissajous systems on the sphere Eur. Phys. J. Plus 129164

[11] Celeghini E, Kuru Ş, Negro J and del Olmo M A 2013 A unified approach to quantum and classical TTW systems based on factorizations Ann. Phys. 33227

[12] Cosgrove C M 2000 Higher-order Painlevé equations in the polynomial class I. Bureau symbol B2 Stud. Appl. Math. 1041

[13] Kudryashov N A 1997 The first and second Painlevé equations of higher order and some relations between them Phys. Lett. A 22435

[14] Hietarinta J 1984 Classical versus quantum integrability J. Math. Phys. 251833

[15] Marquette I 2009 Superintegrability with third order integrals of motion, cubic algebras, and supersymmetric quantum mechanics. II. Painlevé transcendent potentials J. Math. Phys. $\mathbf{5 0} 095202$ 